\title{
News from the San Antonio Breast Cancer Symposium 2017
}

\author{
Chair: \\ Michael Untch ${ }^{\mathrm{a}}$ \\ Participants: Thorsten Kühn ${ }^{b}$ Cornelia Liedtke ${ }^{c}$ Diana Lüftner ${ }^{d}$ Marc Thille

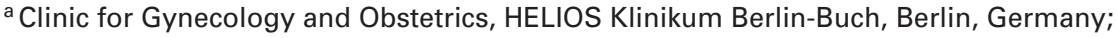 \\ ${ }^{b}$ Department of Gynecology, Esslingen Clinics a.N., Esslingen, Germany; \\ c Department of Gynecology and Breast Centre, Charité University Hospital, Campus Mitte, Berlin, Germany; \\ ${ }^{\mathrm{d}}$ Department of Hematology, Oncology and Tumour Immunology, Charité University Hospital, Campus Benjamin Franklin, Berlin, Germany; \\ e Department of Gynecology and Obstetrics, Agaplesion Markus Krankenhaus, Frankfurt/M., Germany
}

\section{Question 1: Should patients with human epidermal growth factor receptor 2(HER2)-positive or triple- negative breast cancer (TNBC) be treated with neoadjuvant therapy regardless of tumor size or clinical or sonography axillary nodal status?}

Kühn: We treat patients with a low tumor burden (cT1b (i) to small cT1c (i)) with primary surgery. This allows a targeted resection of the original tumor bed without any compromise to the aesthetic outcome. Since clips can migrate in a certain number of cases, primary surgery is associated with more advantages than disadvantages. We can furthermore de-escalate systemic treatment in these patients (omission of anthracyclines and pertuzumab in HER2 and omission of carboplatin in TNBC).

Liedtke: It is (or at least should be) common practice that patients in whom an indication for systemic chemotherapy is recognized at the time of diagnosis this should be given as neoadjuvant chemotherapy in order to assess tumor response and guide additional therapeutic decisions. I would in fact render this more precisely: in patients who have a clear indication for systemic chemotherapy (i.e. aggressive tumor biology) and in whom the optimal chemotherapy regimen is identifiable, patients should be treated with neoadjuvant chemotherapy. This in fact leads to the two limitations of the above statement:

1) Tumor prognosis is determined by tumor biology and tumor stage. In cases with aggressive tumor biology but small tumor stage (i.e. small tumor size and node negativity), the role of chemotherapy is not completely understood. Hence, validation of small tumor size through surgery might be a valuable option in these cases.

2) A limited number of clinical trials has suggested de-escalation of chemotherapy particularly in cases with HER2-positive breast cancer (Tolaney et al., ASCO 2017). These regimens have not been established for the neoadjuvant setting, and inclusion criteria (such as pathological tumor (pT) stage) are solely identifiable through surgery.

Finally, identification of pathologic complete response (pCR) after neoadjuvant chemotherapy is not of equal value among subtypes. For instance, among patients with hormone receptor(HR)positive/HER2-positive breast cancer, patient prognosis is not significantly determined by pCR.

Lüftner: Based on the current data set, these two high-risk entities should be treated with neoadjuvant chemotherapy. Very rarely, patients with node-negative HER-2/neu-positive tumors of less than $3 \mathrm{~cm}$ in diameter can be exempted from this rule where the adjuvant treatment with paclitaxel weekly plus 1 year trastuzumab has become standard of care [1].

Thill: Of course, we derive explicit advantages from treating patients with TNBC and HER2-positive breast cancer with neoadjuvant therapy, such as an in vivo chemosensitivity test to adjust the treatment or a tumor shrinkage to increase the breast conservation rate. Therefore, we state in our current German AGO (Arbeitsgemeinschaft Gynäkologische Onkologie) treatment recommendations: 'If chemotherapy is indicated due to tumor biology, consider systemic treatment before surgery.' However, the decision to give

\section{KARGER}

() 2018 S. Karger GmbH, Freiburg

Fax +497614520714 
chemotherapy depends on tumor biology and tumor size. For patients with HER2-positive tumors I recommend to use a HER2-directed treatment consisting of chemotherapy and trastuzumab plus pertuzumab for tumors sized $>5 \mathrm{~mm}$, regardless of axillary status. The question if tumors of $5 \mathrm{~mm}$ or less should be treated with a monoclonal antibody and chemotherapy has to be discussed on an individual basis. This recommendation goes along with our AGO and the NCCN (National Comprehensive Cancer Network) guidelines. TNBC is commonly known to be an aggressive subtype; nevertheless, not all patients should be treated with chemotherapy. Tumors with a size of $<5 \mathrm{~mm}$ and without nodal involvement do not benefit from chemotherapy; however, if nodal involvement is diagnosed, chemotherapy can be considered. For tumors $>5 \mathrm{~mm}$, I recommend using chemotherapy.

\section{Question 2: Should double anti-HER2 blockade with trastuzumab and pertuzumab be used in HER2-positive breast cancer as neoadjuvant or as adjuvant treatment?}

Kühn: Anti-HER2 systemic treatment with dual blockade should preferably be given as neoadjuvant treatment. This procedure provides important prognostic information and can reduce the extent of surgery.

Liedtke: An easy answer would be to state that currently there is no registration in the adjuvant setting in Europe (expected mid2018). However, given that the adjuvant APHINITY trial has seen a significant but less relevant increase of disease-free survival (DFS) among patients with low-risk HER2-positive disease, I see yet a limited role of adjuvant trastuzumab/pertuzumab among patients with HER2-positive breast cancer.

Lüftner: Neoadjuvant dual anti-HER2 blockade is a standard of care. Data on adjuvant treatment with trastuzumab and pertuzumab after pre-exposure in the neoadjuvant setting is missing, which applies to the majority of patients. Thus, further clinical trials are needed to test the efficacy of this combination in patients with pre-exposure with or without a PCR. In untreated patients, the combination of trastuzumab plus pertuzumab in addition to chemotherapy has shown a slight benefit in terms of invasive DFS with a focus on node-positive patients [2].

Thill: In the neoadjuvant NEOSPHERE trial, the dual blockade of trastuzumab and pertuzumab led to a significant higher pCR compared to trastuzumab alone. In a later analysis, the advantage of having a pCR could be transferred to a survival benefit. Therefore, it is a standard treatment in HER2-positive early breast cancer. Although only $30 \%$ of the patients in the NEOSPHERE trial had been node-negative, I treat all of my patients with the dual blockade of trastuzumab and pertuzumab where a HER2-directed treatment is indicated. A positive HER2 status is enough risk for me.
In the APHINITY trial, the dual blockade of trastuzumab and pertuzumab was shown to have a significant impact on DFS not in the node-negative but in the node-positive as well as in the HR-negative patient population. The difference in DFS was 2.2 and $2.3 \%$, respectively. On the basis of this data, I recommend to treat only high-risk patients with a dual blockade in the adjuvant setting. This is in line with the 7-year follow-up of the APT trial where 12 cycles of paclitaxel and trastuzumab for 1 year led to an excellent survival of $93.3 \%$. In this trial, all patients were nodenegative, $91 \%$ had a tumor size less than $2 \mathrm{~cm}$, and $67 \%$ were HR-positive. To summarize: dual blockade in the neoadjuvant setting for all and in the adjuvant setting for the high-risk population.

\section{Question 3: What do you recommend for residual tumor in the breast or in the axilla after neoadjuvant therapy (for TNBC, for HER2-positive, and for luminal B cancer)?}

Kühn: For patients with HER2-negative disease, additional treatment with capecitabine (according to CREATE-X) may be considered. Since only one randomized trial from Asia is available, confirmatory data are urgently required.

Liedtke: Among patients with HER2-positive disease and residual tumor cells in the breast/axilla after neoadjuvant chemotherapy, there is no established treatment option in addition to trastuzumab yet. If neratinib will be licensed in the nearer future (probably mid/end-2018), this agent might provide a valid option in the case of neoadjuvant treatment resistance. Importantly, the adjuvant ExteNET study has shown efficacy through the addition of sequential neratinib particularly in HR-positive tumors [3]. Among these patients, however, pCR is less relevant as an endpoint since endocrine adjuvant therapy may counterbalance the prognostic value of non-pCR among these patients.

In patients with TNBC, there is only data from one clinical trial that has demonstrated an improvement in prognosis through the addition of capecitabine in patients with non-pCR. However, there are limitations such as toxicity and the fact that the Asian population of this trial is known to show an altered capecitabine metabolism. In case of a BRCA-1/2 mutation, inclusion in the adjuvant OlympiA trial (olaparib adjuvant therapy) represents an important option.

Lüftner: Inclusion into clinical trials and opening of registries for these high-risk patients must be recommended. We currently have the level of evidence IA data supporting any other chemotherapy or biological treatment.

Thill: For TNBC without a pCR after neoadjuvant therapy, we currently do not have post-neoadjuvant treatment options outside of clinical trials. If TNBC is associated with a BRCA mutation, I recommend the participation in the OlympiA study where the 
PARP inhibitor olaparib is used as a maintenance therapy for 12 months. In HER2-positive breast cancer we still wait for the results of the KATHERINE trial and the post-neoadjuvant use of T-DM1 in patients with no pCR. However, the results of the ExteNET study have already been published and have shown a significant improvement of the DFS with an additional use of neratinib for 1 year, an irreversible tyrosine kinase inhibitor of HER1, HER2, and HER4, after 1 year of trastuzumab. However, neratinib is associated with diarrhea that occurred in $40 \%$ with grade 3 within the trial. At the San Antonio Breast Cancer Symposium (SABCS) 2017, the 5-year analysis was presented and showed an advantage in DFS, especially for triple-positive breast cancers, of $4.4 \%$ with a hazard ratio of 0.60 . Therefore, it is a pertinent treatment option. Regarding luminal B-like cancer, the PENELOPE study that was recently closed for recruitment evaluated the use of the combination of the CDK4/6 inhibitor palbociclib in combination with endocrine treatment in high-risk patients without pCR. Let me summarize: Olaparib is an option for patients with BRCA mutation within the OlympiA trial, neratinib is an option in HER2-positive, HR-positive patients but is not yet approved in Europe, and CDK4/6 inhibitors may be used within clinical trials but results are pending.

\section{Question 4: Should patients with histologically proven axillary nodal involvement who have a pCR after neoadjuvant therapy still receive adjuvant radiotherapy (thoracic wall and/or regional lymph nodes)?}

Kühn: Although convincing retrospective data from NSABP B27 are available, suggesting that the response to neoadjuvant chemotherapy provides important information to select patients with a $\mathrm{cN} 1$ status who do not require irradiation of the chest wall after neoadjuvant chemotherapy, these data need to be confirmed in prospective trials. NSABP B51 will provide the definite answer to this question. While awaiting these results, the omission of radiotherapy can be discussed with the patient on an individual basis. Potential risks and benefits must be weighed out. This relates especially to patients scheduled for primary reconstruction.

Liedtke: Although I assume that patients undergoing axillary conversion through neoadjuvant chemotherapy will not benefit from adjuvant radiotherapy to the thoracic wall/regional lymph nodes, there is no clinical trial yet that has shown non-superiority in the case of its omission. Patients should be counselled about this lack of data, particularly in cases where adjuvant radiotherapy leads to increased toxicity (such as in patients with immediate breast reconstruction).

Lüftner: I would like to pass this question on to a radiotherapist.

Thill: It is an important question whether radiation is needed in patients who received a $\mathrm{PCR}$ after neoadjuvant therapy, especially against the background of the association between PCR and a better DFS. Regarding radiation of the thoracic wall after pCR we only have retrospective data that show a significant benefit in lowering the local recurrence rate only for stage I and II disease. Regarding radiation of the regional lymph nodes we also have only retrospective but more inconsistent data. One caveat is the low rate of histologically proven nodal involvement. For radiation of the breast and regional lymph nodes after breast-conserving surgery we also have inconsistent data. In a retrospective analysis of the NSABP B18/27 study, the authors stated that age, clinical-pathologic characteristics before neoadjuvant chemotherapy, and response to chemotherapy in the breast and nodes can be used to optimize radiotherapy. However, mainly stage I and II were included in the trial, axillary staging was done via palpation, and proper information of the HR/HER2 receptor status was missing quite often. Currently, we do not have the data to broadly decrease the use of locoregional radiotherapy after pCR outside of clinical trials. We do have results from several clinical trials that are generating hypotheses how to individualize radiotherapy and how to develop predictive models to decrease or avoid radiotherapy, and quite a few studies are ongoing at the moment.

\section{Question 5: Should patients with metastatic HR-positive, HER2-negative breast cancer without visceral crisis be treated with a combination of endocrine therapy and CDK4/6 inhibitors?}

Kühn: Most patients with estrogen receptor(ER)-positive, HER2-negative metastatic disease derive benefit from the treatment with CDK4/6 inhibitors. The progression-free survival (PFS) is significantly improved (almost 1 year), and most trials demonstrated an improved quality of life. However, the cost-benefit ratio appears to be critical. This issue clearly shows the widening gap between high and low resource countries and the increasing disparities in the worldwide availability of optimal breast cancer care.

Liedtke: If I could provide an answer to this question, I would have probably found the Holy Grail that all of us are currently looking for. It is obvious that not all patients benefit equally; however, there is yet no established clinical/molecular parameter suggesting a subgroup that should or should not be treated with CDK4/6 inhibition. At SABCS 2017, Goetz et al. presented results of a combined analysis of the Monarch-2/-3 study and identified patients with hepatic metastases, low tumor grade, low progesterone receptor expression, and low performance status as deriving particular benefit from abemaciclib therapy. However, these results are in contrast to analyses from the PALOMA and MONALEESA trials who have failed to identify a valid biomarker.

Lüftner: At SABCS 2017, the excellent data of the MONALEESA-7 study were presented; these data supplement all 
other results on postmenopausal patients with metastatic HR-positive, HER2/neu-negative breast cancer. The trial compared endocrine therapy of choice (tamoxifen or letrozole or anastrozole) plus GnRH (gonadotropin-releasing hormone) analog plus/minus ribociclib in premenopausal patients with metastatic HR-positive, HER2/neu-negative breast cancer. Median PFS was 23.8 months in the ribociclib arm compared with 13.0 months in the placebo arm. The overall response rate was significantly higher among patients with measurable disease at baseline in the ribociclib arm compared with the placebo arm (51 vs. $36 \%$ ). Grade 3/4 neutropenia was found in $61 \%$ of patients in the ribociclib arm compared with $4 \%$ in the placebo arm, but it was asymptomatic in most patients. In addition, patients in the ribociclib arm profited in terms of a prolongation until deterioration of quality of life. Thus, the combination with ribociclib must be warranted in this high-risk population [4].

Thill: Currently, three CDK4/6 inhibitors are approved in the USA: palbociclib, ribociclib, and abemaciclib. Palbociclib and ribociclib are also approved in Europe. All compounds have shown a significant improvement in PFS in combination with an aromatase inhibitor (AI) or fulvestrant. The benefit was shown in first-line, second-line, endocrine-resistant, pre- and postmenopausal HR-positive patients with hazard ratios of more or less around 0.55 . We do not know the best treatment sequence, but it is also proven that the next following therapy, including chemotherapy, can be postponed and the treatment-free interval can be prolonged. Therefore, the introduction of CDK4/6 inhibitors is a milestone in the treatment of metastatic breast cancer. Naturally, we do not have to treat all of our first-line patients with an endocrine-based treatment consisting of an endocrine compound and a CDK4/6 inhibitor but at the moment we do not really have an idea which patient we are able to spare a CDK4/6 inhibitor. The PFS benefit was shown in both bone-only and visceral metastatic disease. According to the existing and described data, I think that patients with HER2-negative breast cancer without a lethal crisis should be treated with an endocrine-based treatment, including a CDK4/6 inhibitor. The question whether chemotherapy can be replaced by an endocrine-based treatment with a CDK4/6 inhibitor in first line is unanswered yet. In the beginning of 2018, however, the PADMA trial will start to evaluate palbociclib plus an AI versus chemotherapy in first line; moreover, there is the RIBBIT trial where ribociclib plus an $\mathrm{AI}$ is compared to paclitaxel with or without bevacizumab.

\section{Question 6: Do you see a therapeutic window for PARP inhibitors and for T-cell checkpoint inhibitors in patients with metastatic breast cancer?}

Kühn: Two trials (EMBRACA, OlympiAD) showed a clear advantage of PARP inhibitors (olaparib, talazoparib) in patients with metastatic breast cancer and BRCA-1/2 mutations compared to mono-chemotherapy. This new treatment option will certainly modify future treatment decisions. Daily routine will depend on the approval of these promising new drugs. Regarding checkpoint inhibitors we do not yet have a clear indication for their application, although promising data are accumulating.

Liedtke: With regard to PARP inhibition, there are two large clinical trials (OlympiAD (Robertson et al., ASCO 2017) and EMBRACA (Litton et al., SABCS 2017)) yet who have shown a benefit of PARP inhibition through olaparib and talazoparib among patients with hereditary breast cancer. However, whereas in the OlympiAD trial the results were particularly confirmed among patients with TNBC, in the EMBRACA study responses were seen independent from HR status. It will need to be determined whether genetic BRCA-1/2 status or other biomarkers (HRD, somatic BRCA-1/2 status, TNBC) may represent additional biomarkers indicating response to PARP inhibitor therapy.

With regard to T-cell checkpoint inhibition, there is an increasing body of evidence that these agents might represent future therapeutic options for patients with TNBC. Both the increased mutational load and the high medical need have rendered TNBC as an optimal indication for clinical trials. However, it has to be acknowledged that these agents have demonstrated efficacy also among other breast cancer subgroups. At SABCS 2017, Lou and colleagues presented the results from the KEYNOTE-014/PANACEA study which showed efficacy among patients with metastatic HER2-positive breast cancer, which was particularly pronounced in case of PD-L1 positivity. It needs to be demonstrated whether immune checkpoint inhibition will find a niche in an entity that can be successfully treated with HER2-directed agents in most cases.

Lüftner: Based upon clinical trials and due to the fact that European Medicines Agency approvals are pending, I do not see a clearly defined window for both classes of agents. While the median PFS was significantly longer (7.0 vs. 4.2 months; hazard ratio 0.58 for olaparib) compared to a standard-therapy group of the physician's choice (capecitabine, eribulin, or vinorelbine in 21-day cycles) in pretreated BRCA-positive, metastatic breast cancer patients, this benefit is not very convincing from a clinical point of view [5]. Checkpoint inhibitors as a monotherapy are also of limited clinical effectivity with no clear-cut selection parameters (neither PD-L1 expression nor tumor-infiltrating lymphocytes). Results from first-line trials in combination with chemotherapy have to be awaited.

Thill: After several trials in BRCA-positive, metastatic breast cancer patients without a pertinent efficacy, the phase III OlympiAD trial has been presented at last year's annual ASCO meeting. The trial compared olaparib versus chemotherapy of the physician's choice and showed a significantly improved PFS in BRCAmutated, pre-treated metastatic breast cancer patients of 2.8 months and a hazard rate of 0.58 . It was the first positive phase III trial for a PARP inhibitor. At the SABCS 2017, the PARP success story continued with the EMBRACA study in which talazoparib 
was evaluated against chemotherapy of the physician's choice. Talazoparib led to a significantly improved PFS of 3 months and a hazard ratio of 0.54 in a similar BRCA-mutated patient population. Therefore, I definitely think that there is a therapeutic window for metastatic BRCA-mutated breast cancer.

Regarding T-cell checkpoint inhibitors, numerous studies are going on at the moment. The most promising results were shown in metastatic TNBC. The PD-L1 inhibitor atezolizumab, e.g., showed response rates of up to $40 \%$ together with chemotherapy even in the third line. Checkpoint inhibitors are also active as single agents and responders have an excellent survival, but not more than $20 \%$ respond on average. Cancers with a higher mutation load than breast cancer, such as melanoma or lung cancer, have the highest response rates but nevertheless, many studies in early and metastatic breast cancer are currently recruiting to detect the optimal subgroup of patients for checkpoint inhibition. We have to wait for additional results to identify the patients who will benefit most but we definitely have a therapeutic window.

\section{Participants}

Prof. Dr. Thorsten Kühn

Klinik für Frauenheilkunde und Geburtshilfe

Klinikum Esslingen

Hirschlandstraße 97, 73730 Esslingen, Germany

t.kuehn@klinikum-esslingen.de

Prof. Dr. Cornelia Liedtke

Gynäkologie mit Brustzentrum

Charité - Universitätsmedizin Berlin, Campus Charité Mitte

Charitéplatz 1, 10117 Berlin, Germany

cornelia.liedtke@charite.de

Prof. Dr. med. Diana Lüftner

Medizinische Klinik mit Schwerpunkt Hämatologie und Onkologie Charité - Universitätsmedizin Berlin, Campus Benjamin Franklin

Hindenburgdamm 30, 12200 Berlin, Germany

diana.lueftner@charite.de

PD Dr. med. Marc Thill

Klinik für Gynäkologie und Geburtshilfe

AGAPLESION Markus Krankenhaus

Wilhelm-Epstein-Straße 4, 60431 Frankfurt, Germany

marc.thill@fdk.info

\section{References}

1 Tolaney SM, Barry WT, Dang CT, Yardley DA, Moy B, Marcom PK, Albain KS, Rugo HS, Ellis M, Shapira I, Wolff AC, Carey LA, Overmoyer BA, Partridge AH Guo H, Hudis CA, Krop IE, Burstein HJ, Winer EP: Adjuvant paclitaxel and trastuzumab for node-negative, HER2-positive breast cancer. N Engl J Med 2015 372:134-141.

2 von Minckwitz G, Procter M, de Azambuja E, Zardavas $\mathrm{D}$, Benyunes M, Viale G, Suter T, Arahmani A, Rouchet N, Clark E, Knott A, Lang I, Levy C, Yardley DA, Bines J, Gelber RD, Piccart M, Baselga J; APHINITY Steering Committee and Investigators: Adjuvant pertuzumab and trastuzumab in early HER2-positive breast cancer. N Engl J Med 2017;377:122-131.
3 Martin M, Holmes FA, Ejlertsen B, et al; ExteNET Study Group: Neratinib after trastuzumab-based adjuvant therapy in HER2-positive breast cancer (ExteNET): 5-year analysis of a randomised, double-blind, placebo-controlled, phase 3 trial. Lancet Oncol 2017; 18:1688-1700.

4 Tripathy D, Sohn J, Im S-A, Franke F, Bardia A, Harbeck N, Hurvitz S, Chow L, Lee KS, Campos-Gomez S, Villanueva Vazquez R, Jung KH, Colleoni M, Carlson G, Hughes G, Diaz-Padilla I, Germa C, Hirawat S, Lu Y-S: First-line ribociclib vs placebo with goserelin and tamoxifen or a non-steroidal aromatase inhibitor in premenopausal women with hormone receptor-positive, HER2-negative advanced breast cancer: results from the randomized phase III MONALEESA-7 trial. 2017 San Antonio Breast Cancer Symposium. Presentation GS2-05.
5 Robson M, Im SA, Senkus E, Xu B, Domchek SM Masuda N, Delaloge S, Li W, Tung N, Armstrong A, Wu W, Goessl C, Runswick S, Conte P: Olaparib for metastatic breast cancer in patients with a germline BRCA mutation. N Engl J Med 2017;377:523-533. 